\title{
STIFFNESS SYNTHESIS OF A VARIABLE GEOMETRY PLANAR ROBOT
}

\author{
Nabil Simaan and Moshe Shoham \\ Robotics Laboratory, Department of Mechanical Engineering \\ Technion - Israel Institute of Technology \\ Haifa 32000 \\ ISRAEL \\ http://robotics.technion.ac.il \\ nabil@tx.technion.ac.il \\ shoham@tx.technion.ac.il
}

\begin{abstract}
This paper addresses the problem of task-based stiffness synthesis of a variable geometry three DOF (Degrees Of Freedom) planar robot. The synthesis considers the case where the robot has a limited number of free geometric parameters and constant actuator stiffness coefficients. This defines twenty problems of stiffness synthesis, in which, three parameters of the stiffness matrix are controlled according to task requirements. These problems are modeled as systems of polynomials in the free geometric parameters of the robot's base platform. Using Gröbner bases, the solubility of these polynomial systems is characterized. It is shown that arbitrary desired values of the Cartesian stiffness elements $\left(\mathrm{k}_{\mathrm{xx}}\right.$ and $\left.\mathrm{k}_{\mathrm{yy}}\right)$ are unattainable when only the geometry of the base platform is variable. An example of synthesizing three stiffness elements of the planar robot is solved and shown to have at most 48 solutions in the complex plane. In a numerical case study, sixteen real solutions are obtained, of which only eight are nonsingular.
\end{abstract}

Keywords: Parallel robot, Re-configurable, Stiffness Synthesis, Gröbner bases.

\section{Introduction}

Robots are used to perform various tasks involving complex manipulations and interactions with their environment. Consequently, there are inevitable compromises when using a fixed-geometry robot for some tasks. To overcome this problem, the use of variable geometry robots is considered. In particular we concentrate here on variable geometry parallel robots. These robots can change the geometry of their base/moving platforms to accommodate the required characteristics, e.g. stiffness, specific to each task.

Researchers used various methods to enhance parallel robots' capabilities for better fitting task requirements in terms of stiffness, singularity avoidance, and inclusion of specific paths in the workspace. 
Actuation redundancy was used by Yi and Freeman (1993), Kim et al. (2000) for stiffness modulation. Kinematic redundancy of the robot for a given task was used by Merlet, et al. (2000) for path inclusion in the workspace and singularity avoidance. Stiffness/compliance synthesis algorithms were presented by Huang and Schimmels, (1998), and Roberts, (1999) for a system of springs supporting a rigid body.

Works directly addressing variable geometry parallel robots are limited in number. Zhiming and Song (1998) investigated the design aspects of modular Stewart-Gough platforms with workspace and joint limits considerations. Zhiming and Zhenqun (1999) presented an algorithm for identifying the parameters of the joint locations on the base in a modular Stewart-Gough platform. Merlet, $(1997,2000)$ presented a design algorithm for achieving a constant-orientation workspace of Stewart-Gough robots, which can be adapted for workspace modification of variable geometry robots ${ }^{\star}$.

In the present investigation, a case study of stiffness synthesis for a point in a given path of a planar 3 DOF robot with a variable geometry of its base platform is presented. The aim of the synthesis is to obtain a specific stiffness in a given position/orientation of the robot's moving platform. Under a simplifying assumption that the stiffness coefficients of the redundant actuators that change the base geometry are considerably larger than the coefficients of the other actuators, this work may be viewed as an algorithm for changing the geometry of the base platform of a variable geometry 3 DOF planar robot for obtaining a required stiffness in a point along a path specified by the given task.

\section{Variable Geometry Planar Robot}

The planar robot of Fig. 1 has an equilateral triangular moving platform connected to a circular base by three kinematic chains composed of a slider on the circular base, a revolute joint, a prismatic joint, and a revolute joint on its moving platform. The sliders on the circular base control the geometry of the base platform and the prismatic actuators manipulate the moving

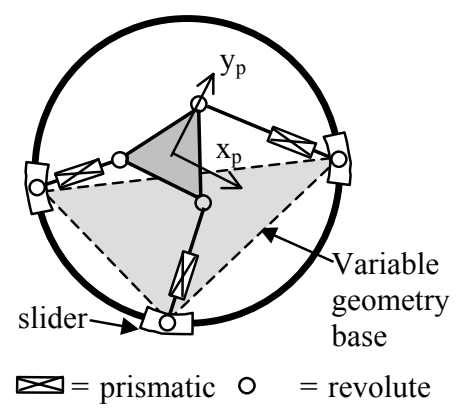

Figure 1. Planar Robot with variable geometry base platform platform. This introduces a kinematic redundancy in this three DOF planar robot. The objective of this paper is to determine the geometry of

\footnotetext{
^ The authors acknowledge the valuable discussions with J.-P. Merlet on this subject
} 
the base platform by computing the locations of these three redundant sliders for achieving a desired stiffness goal in a point of a path defined by the task.

\section{Stiffness Polynomial Formulation}

In this section, the stiffness matrix of the robot of Fig. 1 is formulated as a function of the positions of the sliders on the circular base and is described in a Platform-attached Coordinate System (PCS). The location and orientation of the moving platform are given by the task while the orientations of the prismatic actuators are given by the stiffness synthesis solution and are easily transformed to desired positions of the redundant sliders on the circular base.

The transformation of the desired stiffness from World Coordinate System (WCS) to PCS is given by, Tsai (1999):

$$
{ }^{\mathrm{p}} \mathbf{K}_{\text {des }}=\mathbf{A}^{\mathrm{T} \text { w }} \mathbf{K}_{\text {des }} \mathbf{A} \quad \mathbf{A}=\left[\begin{array}{ll}
\mathbf{R} & \mathbf{0} \\
\mathbf{0} & \mathbf{R}
\end{array}\right]
$$

where $\mathbf{R}$ is the $3 \times 3$ rotation matrix from the PCS to WCS, ${ }^{\mathrm{p}} \mathbf{K}_{\text {des }}$ and ${ }^{\mathrm{w}} \mathbf{K}_{\text {des }}$ are the desired stiffness matrix in PCS and WCS, respectively. Hereafter, all vectors and matrices are represented in PCS.

The only controllable geometric variables by the sliders' locations are the unit vectors of the prismatic actuators' axes, $\left(\hat{\mathbf{l}}_{i}, \hat{i}=1,2,3\right)$, Figs. 1-2.

$$
\hat{\mathbf{i}}_{\mathrm{i}}=\mathrm{a}_{\mathrm{i}} \hat{\mathbf{e}}_{\mathrm{i} 1}+\mathrm{b}_{\mathrm{i}} \hat{\mathbf{e}}_{\mathrm{i} 2} \quad \mathrm{i}=1,2,3
$$

where the symbol $\wedge$ indicates a unit vector, $\hat{\mathbf{e}}_{i 1}$ and $\hat{\mathbf{e}}_{\mathrm{i} 2}$ are unit vectors indicated in Fig. 2, $\hat{\mathbf{i}}_{\mathrm{i}}$ is a unit vector along the $\mathrm{i}^{\text {th }}$ prismatic actuator, and $a_{i}, b_{i}$ are the projections of $\hat{\mathbf{l}}_{\mathrm{i}}$ on $\hat{\mathbf{e}}_{i 1}$ and $\hat{\mathbf{e}}_{\mathrm{i} 2}$. In order for $\hat{\mathbf{l}}_{\mathrm{i}}$ to be a unit vector, $a_{i}$ and $b_{i}(i=1,2,3)$ must obey:

$$
a_{i}^{2}+b_{i}^{2}+2 a_{i} b_{i}\left(\hat{\mathbf{e}}_{i 1} \cdot \hat{\mathbf{e}}_{i 2}\right)-1=0
$$

For an equilateral platform, Eq. (3) is an ellipse in $a_{i}-b_{i}$ plane:

$$
a_{i}^{2}+b_{i}^{2}+a_{i} b_{i}-1=0 \quad i=1,2,3
$$

A simplifying assumption is made that the sliders on the circular platform have a mechanical means to lock rigidly on the circular base once the desired geometry of the base is obtained or that the stiffness coefficients of the sliders are considerably larger than the stiffness coefficients of the prismatic actuators. With this simplifying assumption, the stiffness matrix depends only on the stiffness coefficients of the three remaining active prismatic

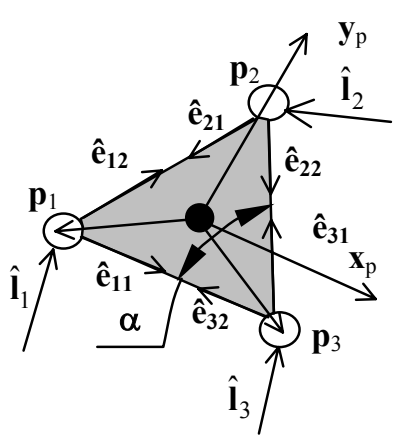

Figure 2. Geometric notations for the planar robot 
actuators. These three active actuators are assumed identical having stiffness coefficient $\mathrm{k}_{\mathrm{d}}$. The rows of the Jacobian matrix of the robot of Fig. 1, with its sliders locked on the circular base, are the Plücker line coordinates of the three axes of the prismatic actuators, Eq. (5).

$$
\mathbf{J}=\left[\begin{array}{cccccc}
\mathrm{a}_{1}+\mathrm{b}_{1} / 2 & \mathrm{~b}_{1} \sqrt{3} / 2 & 0 & 0 & 0 & \mathrm{r} / 2\left(\mathrm{a}_{1}+\mathrm{b}_{1} / 2\right)-3 / 4 \mathrm{rb}_{1} \\
-\mathrm{a}_{2} / 2+\mathrm{b}_{2} / 2 & -\mathrm{a}_{2} \sqrt{3} / 2-\mathrm{b}_{2} \sqrt{3} / 2 & 0 & 0 & 0 & -\mathrm{r}\left(-\mathrm{a}_{2} / 2+\mathrm{b}_{2} / 2\right) \\
-\mathrm{b}_{3}-\mathrm{a}_{3} / 2 & \mathrm{a}_{3} \sqrt{3} / 2 & 0 & 0 & 0 & \mathrm{r} / 2\left(-\mathrm{a}_{3} / 2-\mathrm{b}_{3}\right)+3 / 4 \mathrm{ra}_{3}
\end{array}\right]
$$

The $6 \times 6$ Stiffness matrix of the planar robot with three identical prismatic actuators is given by $\mathbf{K}=\mathrm{k}_{\mathrm{d}} \mathbf{J}^{\mathrm{T}} \mathbf{J}$, Tsai (1999), and the reduced planar $3 \times 3$ stiffness matrix is then, obtained. Notations of the elements of this symmetric stiffness matrix are given in Eq. (6).

$$
\mathbf{K}=\left[\begin{array}{lll}
\mathrm{k}_{\mathrm{xx}} & \mathrm{k}_{\mathrm{xy}} & \mathrm{k}_{\mathrm{x} \theta} \\
\mathrm{k}_{\mathrm{xy}} & \mathrm{k}_{\mathrm{yy}} & \mathrm{k}_{\mathrm{y} \theta} \\
\mathrm{k}_{\mathrm{x} \theta} & \mathrm{k}_{\mathrm{y} \theta} & \mathrm{k}_{\theta \theta}
\end{array}\right]
$$

\section{Stiffness synthesis with limited number of variable geometry parameters}

Given a desired triplet of stiffness elements from the upper triangular part of $\mathbf{K}$ in Eq. (6), the problem of stiffness synthesis with limited number of variable geometry parameters deals with finding the geometry of the base platform (i.e., finding $a_{i}, b_{i}, i=1,2,3$ ) of the robot in Fig. 1.

To fully depict the $3 \times 3$ stiffness matrix, all the six equations in Eq. (7) given below must be fulfilled together with the three equations in Eq (4). Since the planar robot of Fig. 1 has a kinematic redundancy of order three, only three stiffness equations from Eq. (7) can be simultaneously fulfilled.

$$
\mathrm{k}_{\mathrm{n}_{\mathrm{i}}}-\mathrm{k}_{\mathrm{n}_{\text {idesired }}}=0, \mathrm{i}=1,2,3 \quad \mid \quad \mathrm{n}_{1} \neq \mathrm{n}_{2} \neq \mathrm{n}_{3} \in\{\mathrm{xx}, \mathrm{xy}, \mathrm{x} \theta, \mathrm{yy}, \mathrm{y} \theta, \theta \theta\}
$$

Note that this results in $\left(\begin{array}{l}6 \\ 3\end{array}\right)=20$ systems of six polynomials with each having a total degree of 2 in $a_{i}, b_{i}(i=1,2,3)$.

Generally, we ask whether it is possible to solve this problem for any such triplet and, if so, is changing the directions of the lines in Fig. 2 enough to allow controlling all such triplets?

To solve the polynomial systems derived from Eq. (4) and Eq. (7), the method of multiplication tables' eigenvalues (see Stetter (1993)) is used. The following sub-section briefly describes this method. Further details can be found in Möller and Stetter (1995) and Cox et al. (1998). 


\section{The method of eigenvalues for solving polynomial systems}

Let $\mathscr{C}\left[\mathrm{x}_{1} . . \mathrm{x}_{\mathrm{m}}\right]$ represent the ring of polynomials with variables $\mathrm{x}_{1} . . \mathrm{x}_{\mathrm{m}}$, and coefficients over the complex field, $\mathscr{C}$. Let also $S=\left\{p_{1}, p_{2}, . . p_{n} \mid\right.$ $\left.\mathrm{p}_{1}, \mathrm{p}_{2} . . \mathrm{p}_{\mathrm{n}} \in \mathscr{C}\left[\mathrm{x}_{1} . . \mathrm{x}_{\mathrm{m}}\right]\right\}$ be a system of $\mathrm{n}$ polynomials with a corresponding zero-dimensional Ideal $\mathscr{I}=\left\langle\mathrm{p}_{1}, \mathrm{p}_{2}, . . \mathrm{p}_{\mathrm{n}}>, \mathscr{I} \subset \mathscr{C}\left[\mathrm{x}_{1} . . \mathrm{x}_{\mathrm{m}}\right]\right.$. The variety $\mathrm{V}(\mathscr{A})$ of solution is defined by all the $\mathrm{m}$-tuples of $\mathrm{x}_{1} . . \mathrm{xm}$ such that $\mathrm{p}_{1}=\mathrm{p}_{2}=. . \mathrm{p}_{\mathrm{n}}=0$, i.e., $\mathrm{V}(\mathscr{A})=\left\{\left[\mathrm{x}_{1} . . \mathrm{x}_{\mathrm{m}}\right] \in \mathscr{C}^{\mathrm{m}} \mid \mathrm{p}_{1}=\mathrm{p}_{2}=. . \mathrm{p}_{\mathrm{n}}=0\right\}$. We seek all the solutions of $\mathrm{S}$.

The original system of polynomial equations, $\mathrm{S}$, can be replaced by another minimal set of polynomials, $\mathrm{G}=\left\{\mathrm{g}_{1} \ldots \mathrm{g}_{\mathrm{t}}\right\}$, called standard basis (or Gröbner basis) of the ideal $\mathscr{I}$ using Buchberger's algorithm, Buchberger (1965), which is not reviewed here for lack of space. Questions regarding ideal-membership of a given polynomial to $\mathscr{I}$, solubility of $\mathrm{S}$, and finiteness of the dimension of $\mathrm{V}(\mathscr{A})$ are readily answered when using this basis, Heck (1997). Also, for lexographic ordering $G$ is a system of polynomials with successively eliminated number of variables as in the result of Gauss-Jordan elimination method for linear equations. However, this elimination method is unfavorable for large systems due to the computation effort associated with this ordering, Cox et al. (1998).

Two polynomials $\mathrm{f}, \mathrm{g} \in \mathscr{C}\left[\mathrm{x}_{1} . . \mathrm{x}_{\mathrm{m}}\right]$, are said to be congruent modulo $\mathscr{I}$ , $\mathrm{f} \equiv \mathrm{g} \bmod \mathscr{I}$, if $\mathrm{f}-\mathrm{g} \in \mathscr{I}$. Consequently, $\mathrm{f}$ and $\mathrm{g}$ have the same normal form with respect to $G$ and equal cosets $[\mathrm{g}]=[\mathrm{f}]$. A coset $[\mathrm{f}]$ of $\mathrm{f} \in \mathscr{C}\left[\mathrm{x}_{1} . . \mathrm{x}_{\mathrm{m}}\right]$ is the sub-group of $\mathscr{C}\left[\mathrm{x}_{1} . . \mathrm{x}_{\mathrm{m}}\right]$ in which all its elements have the same normal form with respect to $\mathrm{G}$, $[\mathrm{f}]=\mathrm{f}+\mathscr{I}=\{\mathrm{f}+\mathrm{h} \mid \mathrm{h} \in \mathscr{T}\}$. The totality of cosets of the polynomials in $\mathscr{C}\left[\mathrm{x}_{1} . . \mathrm{x}_{\mathrm{m}}\right]$ is the quotient ring of $\mathscr{C}\left[\mathrm{x}_{1} . . \mathrm{x}_{\mathrm{m}}\right]$ modulo $\mathscr{I}$

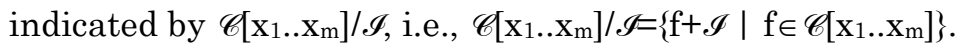

Given two polynomials $\mathrm{f}, \mathrm{g} \in \mathscr{C}\left[\mathrm{x}_{1} . . \mathrm{x}_{\mathrm{m}}\right]$ then a normal form arithmetic similar to number arithmetic exists for addition and multiplication: $\mathrm{n}_{\mathrm{f}}(\mathrm{f}+\mathrm{g})=\mathrm{n}_{\mathrm{f}}(\mathrm{f})+\mathrm{n}_{\mathrm{f}}(\mathrm{g})$ and $\mathrm{n}_{\mathrm{f}}(\mathrm{f} \mathrm{g})=\mathrm{n}_{\mathrm{f}}\left(\mathrm{n}_{\mathrm{f}}(\mathrm{f}) \mathrm{n}_{\mathrm{f}}(\mathrm{g})\right)$. Since every normal form is associated with a coset and vise-versa, this arithmetic is also translated to an associated coset arithmetic in the ring $\mathscr{C}\left[\mathrm{x}_{1} \ldots \mathrm{x}_{\mathrm{m}}\right] / \mathscr{I}$ resulting in the fact that $\mathscr{C}\left[\mathrm{x}_{1} . . \mathrm{x}_{\mathrm{m}}\right] / \mathscr{I}$ is a vector space in $\mathscr{C}^{\mathrm{n}}$. Let $\mathrm{B}$ be a basis of monomials for this space $B=\left\{b_{i}, i=1 . . n\right\}$. This means that the remainder (or normal form) of any $\mathrm{f} \in \mathscr{C}\left[\mathrm{x}_{1} . . \mathrm{x}_{\mathrm{m}}\right]$ is given by:

or in congruence terms: $\quad \mathrm{f} \equiv \sum_{\mathrm{i}=1 . . \mathrm{n}} \mathrm{c}_{\mathrm{i}} \mathrm{b}_{\mathrm{i}} \bmod \mathscr{I}$

Define the monomial basis vector $\mathbf{b}=\left[b_{1}, b_{2}, \ldots b_{n}\right]^{t}, b_{i} \in B, i=1 . . n$, then each polynomial $\mathrm{f} \in \mathscr{C}\left[\mathrm{x}_{1} . . \mathrm{x}_{\mathrm{m}}\right]$ has a multiplication table $\mathbf{M}_{\mathrm{f}}$ such that:

$$
\mathrm{f} \mathbf{b} \equiv \mathbf{M}_{\mathrm{f}} \mathbf{b} \bmod \mathscr{I}
$$


Since the congruence relation in Eq. (10) indicates that $\mathrm{f} \mathbf{b}-\mathbf{M}_{\mathrm{f}} \mathbf{b} \in \mathscr{I}$ then $\mathrm{f} \mathbf{b}-\mathbf{M}_{\mathrm{f}} \mathbf{b}=\mathbf{0}$ for all the points of $\mathrm{V}(\mathscr{A})$. Consequently, the eigenvalues method is based on Eq. (11):

$$
\left(\mathbf{M}_{\mathrm{f}}-\mathrm{f} \mathbf{I}\right) \mathbf{b}=\mathbf{0}
$$

Equation (11) shows that the eigenvalues of $\mathbf{M}_{\mathrm{f}}$ are the values of $\mathrm{f}$ for all the points of $\mathrm{V}(\mathscr{A})$. If $\mathrm{f}$ is taken as $\mathrm{f}=\mathrm{x}_{1}$ then Eq. (11) gives all the coordinates $\mathrm{x}_{1}$ of the points of $\mathrm{V}(\mathscr{A})$. Thus, by constructing the multiplication tables $\mathbf{M}_{\mathrm{x} 1} \ldots \mathbf{M}_{\mathrm{xm}}$ and solving for their eigenvalues, all the values of the coordinates $\mathrm{x}_{1} \ldots \mathrm{x}_{\mathrm{m}}$ for the points of $\mathrm{V}(\mathscr{A})$ are obtained. The minimal polynomials of $\mathbf{M}_{\mathrm{xi}}, \mathrm{i}=1 . . \mathrm{m}$, when written with $\mathrm{x}_{\mathrm{i}}$ as its variable, give the monic generators of the elimination ideals $\mathscr{I} \cap \mathscr{C}\left[\mathrm{x}_{\mathrm{i}}\right]$.

There are both symbolic and numerical advantages of this method compared to standard sequential elimination of variables by resultants, Raghavan and Roth (1995), Neilsen and Roth (1999). Since this method is based on Gröbner basis construction, solvability of the system of polynomial equations is readily determined. Moreover, this method is unaffected by the term order used for the computation of G, which reduces the computation time when using more efficient term orders such as total degree order, Cox et al. (1998). Compared to sequential elimination methods, in this method the numerical computation is kept to a minimum since numerical values are used only in the computation procedure of eigenvalues and the solution of each coordinate $\mathrm{x}_{\mathrm{i}}$ is independent of the numerical solutions of the other variables and, thus, it is unaffected by computation errors in the other variables $\mathrm{x}_{\mathrm{j}}$.

\section{Application to the parallel planar robot}

To answer the questions of section 4, the reduced Gröbner bases associated with all the 20 possible systems of equations in the form of Eq. (7) were computed. A total-degree ordering with $a_{1}>b_{1}>a_{2}>b_{2}>a_{3}>b_{3}$ was used for reducing the computation effort of these bases.

The non-solubility of a polynomial system is determined by checking whether its reduced Gröbner basis is $\{1\}$, Adams and Loustanau (1994). Performing this task using Maple $^{\circledR}$ shows that all the polynomial systems including equations for simultaneously fulfilling the desired values of $\mathrm{k}_{\mathrm{xx}}$ and $\mathrm{k}_{\mathrm{yy}}$ are unsolvable. Consequently, changing the directions of the prismatic actuators relative to the moving platform is not sufficient for simultaneously achieving these stiffness elements.

Next, the problem of Eq. (7) for $\mathrm{k}_{\mathrm{xx}}, \mathrm{k}_{\mathrm{xy}}$, and $\mathrm{k}_{\mathrm{x} \theta}$ is solved, i.e., all the stiffness elements in the $\mathrm{x}$ direction of PCS are prescribed based on task requirements. The reduced Gröbner basis for this problem, hereafter called G, with total degree ordering $a_{1}>b_{1}>a_{2}>b_{2}>a_{3}>b_{3}$ has 28 generators of degrees ranging from 1 to 5 in the variables. The $i^{\text {th }}$ column in table 1 
presents the degrees of the $\mathrm{i}^{\text {th }}$ basis polynomial in the variables corresponding to $\mathrm{a}_{1}, \mathrm{~b}_{1}, \mathrm{a}_{2}, \mathrm{~b}_{2}, \mathrm{a}_{3}, \mathrm{~b}_{3}$. It can be seen that the total degree ranges from 4 to 8 . This basis is not presented here due to lack of space.

Table 1. Degrees of the 28 polynomials of $\mathrm{G}$ in the variables

\begin{tabular}{|c|c|}
\hline $\mathrm{a}$ & {$[0,0,0,0,1,2,1,1,0,1,1,0,0,1,1,0,1,1,1,0,1,0,0,0,0,1,1,1]$} \\
\hline $\mathrm{b}_{1}$ & $0,0,0,2,1,0,1,1,0,1,1,0,0,1,1,0,1,1,1,0,1,0,0,0,0,1,1,1$ \\
\hline $\mathrm{a}_{2}$ & $0,1,2,0,0,0,1,1,1,0,0,1,1,0,0,0,1,1,1,0,1,0,0,1,1,0,0,1$ \\
\hline $\mathrm{b}_{2}$ & $0,2,2,2,2,2,1,1,3,2,2,1,1,0,0,2,1,1,1,2,1,2,2,1,1,0,0,1$ \\
\hline $\mathrm{a}_{3}$ & $2,1,1,0,1,1,1,1,1,1,1,1,1,1,1,1,0,0,0,1,1,1,1,1,1,1,1,1$ \\
\hline $\mathrm{b}_{3}$ & {$[2,2,2,2,2,1,1,1,2,2,2,3,3,3,3,4,2,2,2,4,2,5,4,4,4,4,4,3]$} \\
\hline
\end{tabular}

The leading terms of $\mathrm{G}$ are given by:

$$
\begin{aligned}
& \text { LT }=\left[a_{3}{ }^{2}, a_{2} b_{2}, a_{2} 2, b_{1}^{2}, a_{1} b_{1}, a_{1} 2, a_{3} a_{2} b_{1}, a_{3} a_{1} a_{2}, b_{2} 3, b_{2} b_{1}, b_{2}{ }^{2} a_{1}\right. \text {, } \\
& b_{3} 2 a_{3} b_{2}, b_{3} a_{3} a_{2}, b_{3} 2 b_{1} a_{3}, b_{3} 2 a_{3} a_{1}, b_{2} 2 b_{3} 2, b_{3} b_{2} a_{1}, b_{3} 2 a_{2} b_{1}, b_{3} a_{1} a_{2} \text {, } \\
& \left.b_{2} a_{3} b_{3}, a_{3} b_{2} b_{3} a_{1}, b_{3} 5, b_{3} 4 a_{3}, b_{3} 4 b_{2}, b_{3} 4 a_{2}, b_{3} 4 b_{1}, a_{1} b_{3} 4, b_{3} 3 b_{2} b_{1}\right]
\end{aligned}
$$

Based on the finiteness theorem, Adams and Loustaunau (1994), the system of polynomials corresponding to $\mathrm{G}$ is solvable and has a zerodimensional variety. This is established by examining the group of leading terms in Eq. (12) which shows that each variable among $\left\{\mathrm{a}_{1}, \mathrm{~b}_{1}, \mathrm{a}_{2}, \mathrm{~b}_{2}, \mathrm{a}_{3}, \mathrm{~b}_{3}\right\}$ appears alone as a leading term in $\mathrm{G}$ with the corresponding degrees of $\{2,2,2,3,2,5\}$. Consequently, the group of all the remainders in $\mathscr{C}\left[\mathrm{a}_{1}, \mathrm{~b}_{1}, \mathrm{a}_{2}, \mathrm{~b}_{2}, \mathrm{a}_{3}, \mathrm{~b}_{3}\right] / \mathscr{I}$, denoted by $\mathrm{D}$, has terms with maximal degrees of $\{1,1,1,2,1,4\}$ in $\left\{a_{1}, b_{1}, a_{2}, b_{2}, a_{3}, b_{3}\right\}$, respectively.

The monomial basis of $\mathscr{C}\left[\mathrm{a}_{1}, \mathrm{~b}_{1}, \mathrm{a}_{2}, \mathrm{~b}_{2}, \mathrm{a}_{3}, \mathrm{~b}_{3}\right] / \mathscr{A}$, denoted by $\mathrm{B}$, is found from $\mathrm{D}$ by extracting all the monomials in $\mathrm{D}$ that are equal to their own normal forms, Cox et al. (1998). This leads to the 48-dimensional monomial basis in Eq. (13):

$$
\begin{aligned}
& B=\left[1, b_{3}, a_{3}, b_{2}, a_{2}, b_{1}, a l_{3} b_{3}^{2}, a_{3} b_{3}, b_{2} b_{3}, a_{2} b_{3}, b_{1} b_{3}, a_{1} b_{3}, a_{3} b_{2}, a_{3} a_{2}, a_{3} b_{1}, a_{3} a_{1}\right. \\
& b_{2}{ }^{2}, b_{2} b_{1}, b_{2} a 1, a_{2} b_{1}, a_{1} a_{2}, b_{3} 3, b_{3}^{2} a_{3}, b_{2} b_{3}{ }^{2}, b_{3}{ }^{2} a_{2}, b_{3}{ }^{2} b_{1}, b_{3} 2 a 1, b_{3} a_{3} b_{2} \text {, } \\
& a_{3} a_{2} b_{3}, b_{3} a_{3} b_{1}, a_{3} a 1 b_{3}, b_{3} b_{2}, b_{3} b_{2} b_{1}, b_{3} b_{2} a 1, b_{1} a_{2} b_{3}, a_{1} a_{2} b_{3}, a_{3} b_{2}, a_{3} b_{2} b_{1} \text {, } \\
& \left.a_{3} b_{2} a_{1}, b_{3}{ }^{4}, a_{3} b_{3}, b_{3} b_{2}, a_{2} b_{3}, b_{3} b_{1}, a b_{3}, b_{3} b_{2} b_{1}, b_{1} b_{2} a_{3} b_{3}\right]
\end{aligned}
$$

Next, three $48 \times 48$ multiplication tables, $\mathbf{M}_{\mathrm{f} 1}, \mathbf{M}_{\mathrm{f} 2}$ and $\mathbf{M}_{\mathrm{f} 3}$ for $\mathrm{f}_{1}=\mathrm{a}_{1}+\mathrm{b}_{1}$, $\mathrm{f}_{2}=\mathrm{a}_{2}+\mathrm{b}_{2}, \mathrm{f}_{3}=\mathrm{a}_{3}+\mathrm{b}_{3}$ are computed together with their corresponding minimal polynomials $\mathrm{mp}_{\mathrm{f} 1}, \mathrm{mp}_{\mathrm{f} 2}$, and $\mathrm{mp}_{\mathrm{f} 3}$. These minimal polynomials have only even degrees, so there are at most 24 pairs of complex solutions and their conjugate solutions (48 in total). These solutions give the values of $f_{1}=a_{1}+b_{1}, f_{2}=a_{2}+b_{2}$, and $f_{3}=a_{3}+b_{3}$. The next step is solving for the values of $a_{1}, b_{1}, a_{2}, b_{2}, a_{3}, b_{3}$. These values establish the locations of the sliders on the circular base. The solution algorithms for obtaining the 
values of $\left(a_{1}, b_{1}\right),\left(a_{2}, b_{2}\right)$, and $\left(a_{3}, b_{3}\right)$ are identical; hence, only the algorithm for obtaining $\left(a_{1}, b_{1}\right)$ is presented herein.

Let $\pm \mathrm{C}$ be one of the 24 solution pairs of mpf1. The matching solutions for $\left(a_{1}, b_{1}\right)$ are the intersections of the line and the ellipse of Eq. (14).

$$
\mathrm{a}_{1}+\mathrm{b}_{1}= \pm \mathrm{C} \cap \mathrm{a}_{1}{ }^{2}+\mathrm{b}_{1}{ }^{2}+\mathrm{a}_{1} \mathrm{~b}_{1}-1=0
$$

The solutions for $+\mathrm{C}$ and for $-\mathrm{C}$ are:

$$
\begin{aligned}
& \text { for }+\mathrm{C}:(\mathrm{a} 1, \mathrm{~b} 1)=\left(\frac{1}{2} \mathrm{C} \pm \frac{1}{2} \Delta, \frac{1}{2} \mathrm{C} \mp \frac{1}{2} \Delta\right) \quad \Delta \equiv \sqrt{4-3 \mathrm{C}^{2}} \\
& \text { for }-\mathrm{C}:(\mathrm{a} 1, \mathrm{~b} 1)=-\left(\frac{1}{2} \mathrm{C} \mp \frac{1}{2} \Delta, \frac{1}{2} \mathrm{C} \pm \frac{1}{2} \Delta\right)
\end{aligned}
$$

Since only real solutions for $\left(\mathrm{a}_{1}, \mathrm{~b}_{1}\right)$ are of interest, only the admissible real solution pairs of $\mathrm{mp}_{\mathrm{f} 1}$ satisfying $|\mathrm{C}| \leq 2 / \sqrt{3}$ are used in Eq. (15). Note that the two solutions for $+\mathrm{C}$ (and $-\mathrm{C}$ ) represent a mirror image about the bisector of the angle $\alpha$ in Fig. 2 and that the two solutions for $+\mathrm{C}$ are mirror images of the two solutions for $-\mathrm{C}$ about the normal to the bisector of the angle $\alpha$ in Fig. 2.

Once this procedure is repeated for the roots of $\mathrm{mp}_{\mathrm{f} 2}$ and $\mathrm{mp}_{\mathrm{f} 3}$, sets of solutions for $\left(\mathrm{a}_{1}, \mathrm{~b}_{1}\right),\left(\mathrm{a}_{2}, \mathrm{~b}_{2}\right)$ and $\left(\mathrm{a}_{3}, \mathrm{~b}_{3}\right)$ are obtained and all the sextuplets $\left(a 1, b_{1}, a_{2}, b_{2}, a_{3}, b_{3}\right)$ satisfying Eqs. $(4,7)$ are found.

\section{Numerical Example}

To verify the solution procedure, a predefined geometry of the planar robot was selected with $\left[\mathrm{a}_{1}, \mathrm{~b}_{1}, \mathrm{a}_{2}, \mathrm{~b}_{2}, \mathrm{a}_{3}, \mathrm{~b}_{3}\right]=[\sqrt{3} / 3, \sqrt{3} / 3,1,0,1,0]$. This corresponds to $\left[\theta_{1}, \theta_{2}, \theta_{3}\right]=\left[30^{\circ}, 240^{\circ}, 120^{\circ}\right]$, where $\theta_{1}, \theta_{2}$, and $\theta_{3}$ are the angles of $\hat{\mathbf{i}}_{1}, \hat{\mathbf{i}}_{2}$, and $\hat{\mathbf{i}}_{3}$ relative to $\mathbf{x}_{\mathrm{p}}$ in Fig. 2. The corresponding stiffness matrix, using a platform radius of $0.1[\mathrm{~m}]$ and $\mathrm{k}_{\mathrm{d}}=1 \mathrm{e}+5[\mathrm{~N} / \mathrm{m}]$, is:

$$
\mathrm{K}=\left[\begin{array}{ccc}
125000.00000 & 43301.270189 & -5000.00000 \\
43301.2701 & 174999.99999 & 0 . \\
-5000.00000 & 0 . & 500.00000
\end{array}\right]
$$

The aim of the following example is finding all the solutions for $\left(a_{i}, b_{i}\right)$, $\mathrm{i}=1,2,3$, for obtaining the stiffness elements $\mathrm{k}_{\mathrm{xx}}, \mathrm{k}_{\mathrm{xy}}$, and $\mathrm{k}_{\mathrm{x} \theta}$ of Eq. (16) at a given manipulation point of the path. The solution method is validated if one of the solutions gives the values of the predefined geometry.

Three minimal polynomials, $\mathrm{mp}_{\mathrm{f} 1}, \mathrm{mp}_{\mathrm{f} 2}$, and $\mathrm{mp}_{\mathrm{f} 3}$ are obtained with their solutions. Table 2 lists only the admissible distinct solution pairs $\mathrm{C}_{1}, \mathrm{C}_{2}$, and $\mathrm{C}_{3}$, of $\mathrm{mp}_{\mathrm{f} 1}, \mathrm{mp}_{\mathrm{f} 2}$, and $\mathrm{mp}_{\mathrm{f} 3}$, respectively. These solutions are distinct up to 1e-3 resolution from other close solutions. Table 3 lists the distinct 16 solutions for $a_{i}, b_{i}, i=1,2,3$. Note that, as expected, Table 3 contains a solution corresponding to the exact values of $[\sqrt{3} / 3, \sqrt{3} / 3,1,0,1,0]$ of the pre-defined example. These 16 solutions are presented in Fig. 3. Note also that only the last eight solutions, (i) through (p), are nonsingular. 
Table 2. Admissible real distinct solutions of $\mathrm{mpf}$, mpf2, and mpf3

\begin{tabular}{lllll}
\hline $\mathrm{C}_{1}$ & \pm 0.577350 & $\pm(1-4.4 \mathrm{e}-15)^{*}$ & \pm 1.145112 & \pm 1.154700 \\
$\mathrm{C}_{2}$ & $\pm 0.38207 \mathrm{e}-13$ & \pm 0.376135 & \pm 0.967869 & \pm 0.999999 \\
$\mathrm{C}_{3}$ & \pm 0.514087 & \pm 0.968432 & $\pm(1+12 \mathrm{e}-30)^{*}$ & \pm 1.154700
\end{tabular}

* All numerical computations were made with 32 digits, but results are truncated to 6 decimal digits for presesntation purposes

Table 3. 16 real solutions to the problem in the numerical example

\begin{tabular}{cccccccc}
\hline $\mathrm{a} 1$ & $\mathrm{~b} 1$ & $\mathrm{a} 2$ & $\mathrm{~b} 2$ & $\mathrm{a} 3$ & $\mathrm{~b} 3$ \\
$0.8869987582^{-14}$ & -1 & -1 & 0.33502100 & $10^{-9}$ & \pm 0.57735027 & \pm 0.57735027 \\
$0.8869987582^{-14}$ & -1 & 1 & -0.33502100 & $10^{-9}$ & \pm 0.57735027 & \pm 0.57735027 \\
$-0.8869987582^{-14}$ & 1 & -1 & 0.33502100 & $10^{-9}$ & \pm 0.57735027 & \pm 0.57735027 \\
$-0.8869987582^{-14}$ & 1 & 1 & -0.33502100 & $10^{-9}$ & \pm 0.57735027 & \pm 0.57735027 \\
0.57735027 & 0.57735027 & -1 & 0.33502100 & $10^{-9}$ & \pm 1 & $\pm 0.224010^{-28}$ \\
0.57735027 & 0.57735027 & 1 & -0.33502100 & $10^{-9}$ & \pm 1 & $\pm 0.224010^{-28}$ \\
-0.57735027 & -0.57735027 & -1 & 0.33502100 & $10^{-9}$ & \pm 1 & $\pm 0.224010^{-28}$ \\
-0.57735027 & -0.57735027 & 1 & -0.33502100 & $10^{-9}$ & $\pm 0.224010^{-28}$
\end{tabular}

Figure 3. 16 solutions with only eight non-singular solutions (i, through p)

\section{Conclusions}

This investigation addresses the stiffness synthesis problem of a planar kinematically redundant 3 -DOF robot by controlling a limited number of its free geometric parameters. It is shown that it is impossible to control both Cartesian stiffness elements $\mathrm{k}_{\mathrm{xx}}$ and $\mathrm{k}_{\mathrm{yy}}$, by only changing the locations of the sliders on its circular base. In an example of controlling $\mathrm{k}_{\mathrm{xx}}, \mathrm{k}_{\mathrm{xy}}$, and $\mathrm{k}_{\mathrm{x} \theta}$, it is shown that, at most, there are 48 solutions in the complex plane. The numerical example solved shows only eight real non-singular solutions.

\section{Acknowledgements}

This research has been partially supported by the Israeli Ministry of Science under the French-Israel program. 


\section{References}

Adams W., Loustanau, Ph., (1994), An Introduction to Grobner Bases, Graduate Studies in Mathematics, Vol. 3, American Mathematical Society.

Buchberger, B., (1965), An Algorithm for Finding a Basis for Residue Class Ring of a ZeroDimensional Polynomial Ideals (German). PhD Thesis, University of Innsbruck, Institute for Mathematics.

Cox, D., Little, J., O’Shea, D., (1998), Using Algebraic Geometry, Graduate Texts in Mathematics, no. 185, Springer-Verlag.

Heck, A., (1997), "Bird's-eye View of Grobner Bases," Nuclear Instruments and Methods in Physics Research, vol. A 389, pp. 16-21.

Huang, S., Schimmels, J., (1998), “Achieving an Arbitrary Spatial Stiffness with Springs Connected in Parallel," J. of Mechanical Design, Vol. 120, pp.520-526.

Kim, W. K., Yi, B. J., Cho, W., (2000) "RCC Characteristics of Planar/Spherical Three Degrees of Freedom Parallel Mechanisms with Joint Compliances," ASME Mechanical Design, Vol. 122, pp. 10-16.

Möller H. M., Stetter, H. J., (1995), "Multivariate Polynomial Equations With Multiple Zeros Solved by Matrix Eigenproblems," Numer. Math., no. 70, pp. 311-329.

Stetter, H. J., (1993), "Multivariate Polynomial Equations as Matrix Eigenproblems," In: Contributions in Numerical Mathematics, World Scientific Series in Applicable Analyis (WSSIAA), vol. 2, pp. 355-371.

Merlet, J-P., (1997), “Designing a Parallel Manipulator for a Specific Workspace," Intl. J. of Robotics Research, Vol. 16, No. 4, September, pp. 545-556.

Merlet, J-P., (2000), Parallel Robots, Kluwer Academic publishers.

Merlet, J-P., Perng, M-W., Daney D., (2000), "Optimal Trajectory Planning of a 5-Axis Machine-Tool Based on a 6-Axis Parallel Manipulator," In Advances in Robot Kinematics, Lenarcic, J. and Stanisic M. M., Eds., Kluwer Academic Publishers, pp. 315-322.

Neilsen, J., Roth, B., (1999), "On the Kinematic Analysis of Robotic Mechanisms," Intl. J. of Robotics Research, vol. 18, no. 12, pp. 1147-1160.

Raghavan, M., Roth, B., (1995), "Solving Polynomial Systems for the Kinematic Analysis and Synthesis of Mechanisms and Robot Manipulators," Transactions of ASME, Special $50^{\text {th }}$ Anneversary Design Issue, Vol. 117, pp. 71-79.

Roberts, G. R., (1999), "Minimal Realization of a Spatial Stiffness Matrix with Simple Springs Connected in Parallel," IEEE Transactions on Robotics and Automation, Vol. 15, No.,5.

Tsai, L-W., (1999), Robot Analysis - The Mechanics of Serial and Parallel Manipulators, John Wiley \& Sins, Inc.

Yi, B. Ji. and Freeman, R. A., (1993) "Geometric Characteristics of Antagonistic Stiffness In Redundantly Actuated Mechanisms," IEEE International Conference on Robotics and Automation, pp. 654-661.

Zhiming, J., Song Ph., (1998), “Design of a Reconfigurable Platform Manipulators,” In J. of Robotic Systems, Vol. 15. No. 6, pp. 341-346.

Zhiming, J., Zhenqun, L., (1999), "Identification of Placement Parameters for Modular Platform Manipulators,” In J. of Robotic Systems, Vol. 16. No. 4, pp. 227-236. 